\title{
Far Ultra-Violet Polarimeter by reflection for Pollux (LUVOIR)
}

Maëlle Le Gal, Arturo Lopez Ariste, Martin Pertenais, Coralie Neiner ICSO, October 12th 2018

I'Observatoire LESIA

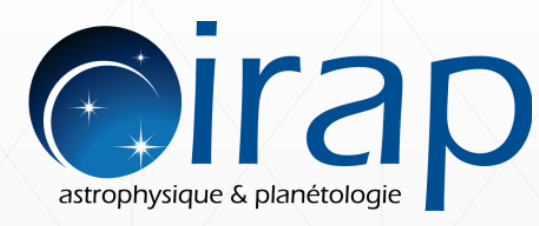

denes

CENTRE NATIONAL D'ÉTUDES SPATIALES

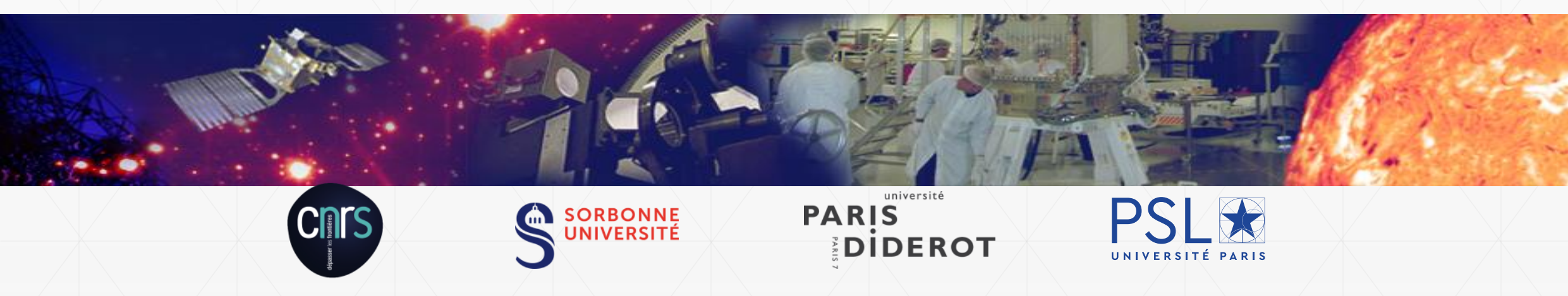




\section{Outline}

- Context: Pollux for LUVOIR

- Reminder

- Principle: temporal modulation

- FUV polarimeter : reflection

- Tests to measure $\mathrm{X}$ and $\tau$

- Conclusion

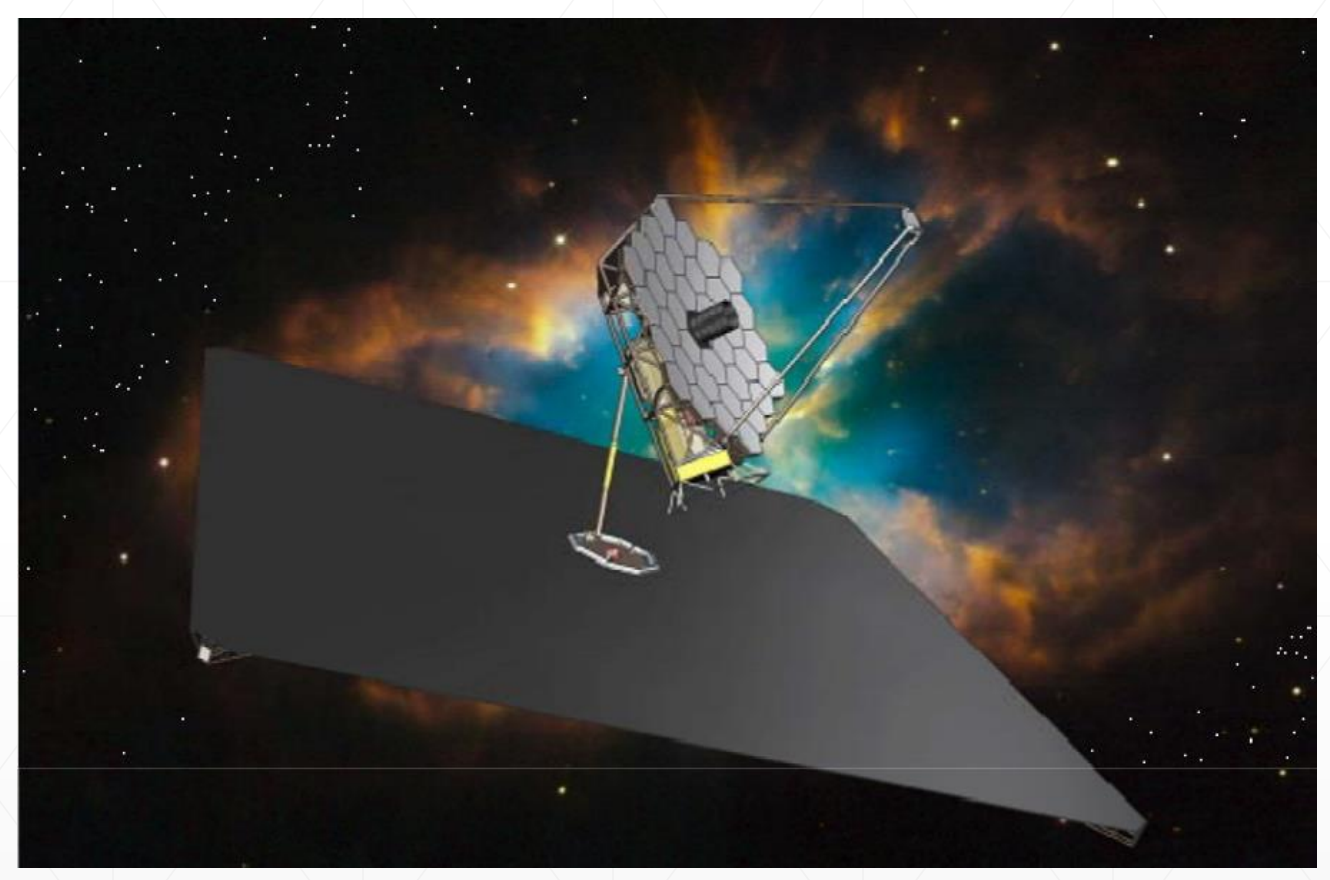




\section{Pollux for LUVOIR}

\section{LUVOIR}

- Large UV/Optical/IR Surveyor: From UV to IR

- NASA Project of a 15-m diameter space telescope

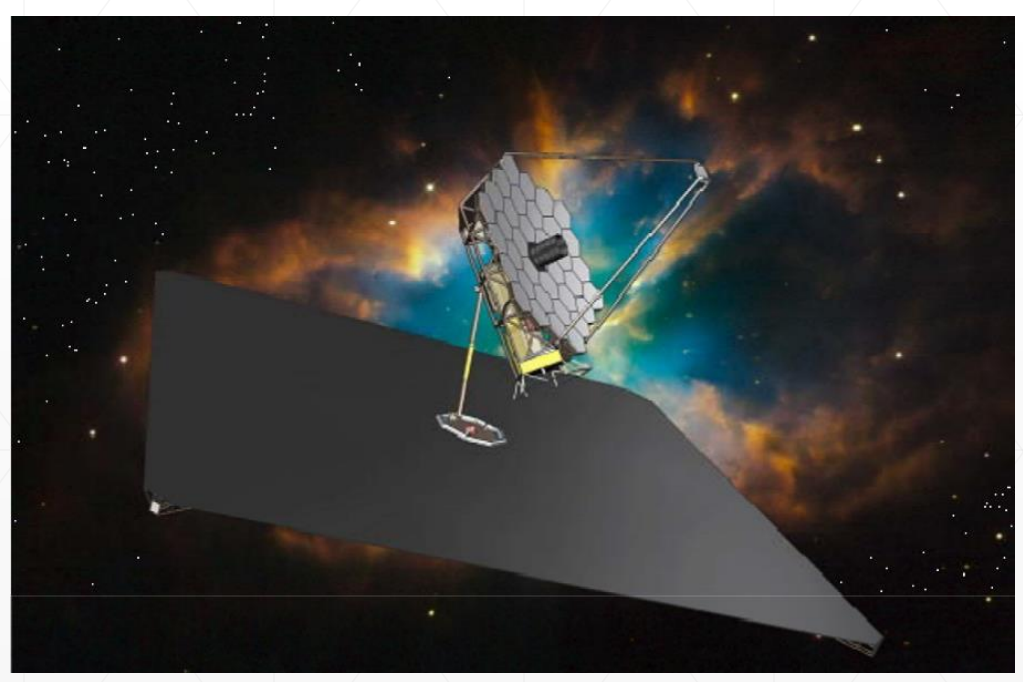

\section{POLLUX}

- High-resolution spectropolarimeter

- Leadership CNES/LESIA/LAM, European consortium

- 3 channels

$\begin{array}{ll}\text { FUV } & 90 \mathrm{~nm}-124.5 \mathrm{~nm} \\ \text { MUV } & 118.5 \mathrm{~nm}-195 \mathrm{~nm} \\ \text { NUV } & 190 \mathrm{~nm}-390 \mathrm{~nm}\end{array}$




\section{Reminder}

- Polarization: geometrical orientation of $\mathrm{E}$

- Projection on the plane orthogonal to the propagation direction: ellipse

- Stokes Vector:

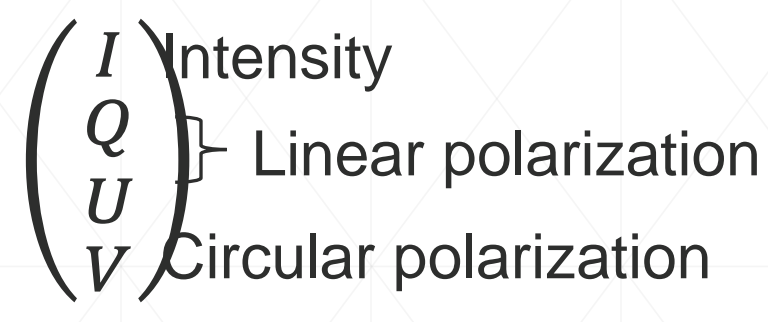

- Mueller Matrix:

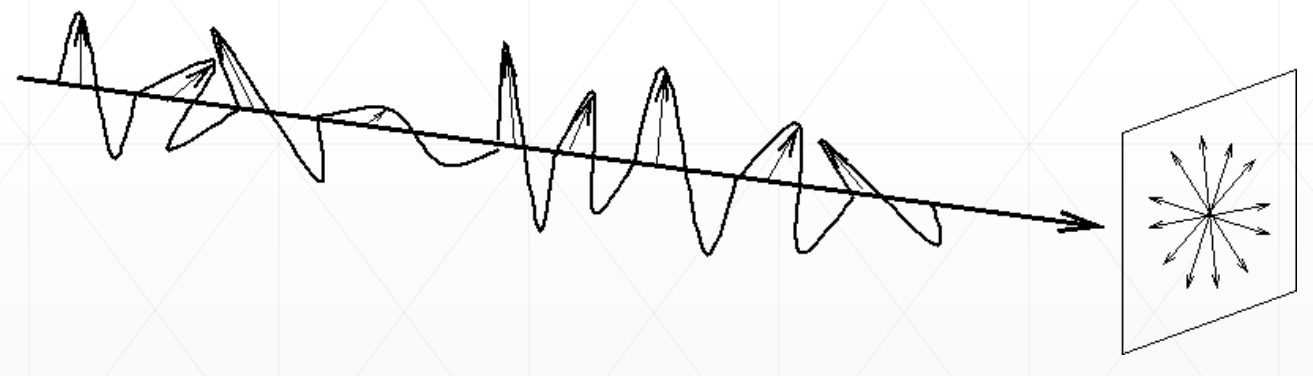

- Characterise a component

- Define polarization modification:

$$
\overrightarrow{S_{\text {out }}}=M \overrightarrow{S_{\text {in }}}
$$

- Spectropolarimetry: measurements of polarization as a function of wavelength 


\section{Polarimeter principle : temporal modulation}

- Rotating modulator (discrete positions)

- Analyzer: fixed linear polarizer
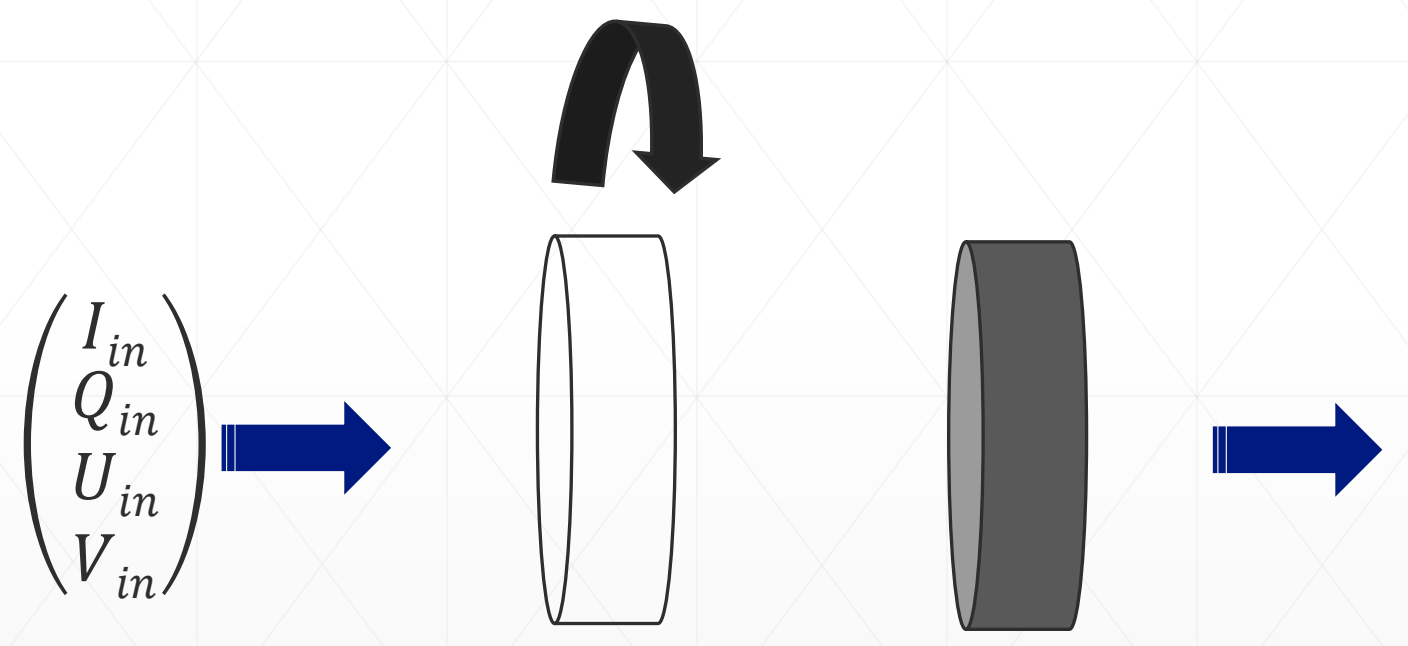

$$
\begin{aligned}
I_{\text {out }}(x, \lambda)= & I_{\text {in }} \\
& +\alpha_{Q}(\lambda) Q_{\text {in }}(\lambda) \\
& +\alpha_{U}(\lambda) U_{\text {in }}(\lambda) \\
& +\alpha_{V}(\lambda) V_{\text {in }}(\lambda)
\end{aligned}
$$

Rotating modulator

Analyzer 


\section{FUV Polarimeter : $90-124.5 \mathrm{~nm}$}

- In FUV, no transmitting birefringent material $\rightarrow$ polarimeter by reflection

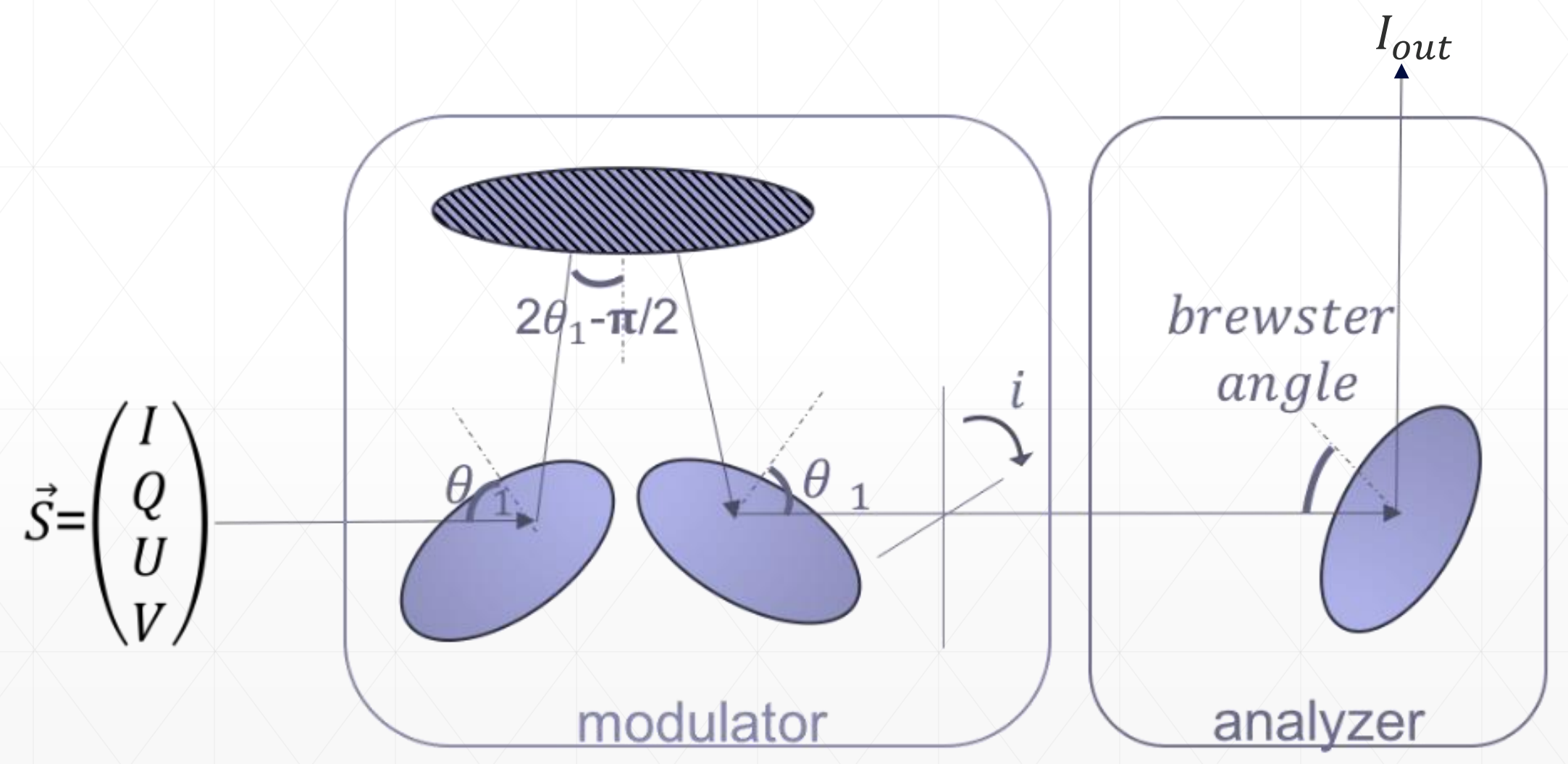




\section{Modulation 0: Measurements $=0$ * Stokes}

- Modulation matrix: product of all the Mueller matrices

$$
O=M_{R}\left(i_{\text {Brewster }}, \lambda\right) * R(-\theta) * M_{R}(i, \lambda) * M_{R}\left(2 i-\frac{\pi}{2}, \lambda\right) * M_{R}(i, \lambda) * R(\theta)
$$




\section{Modulation 0: Measurements $=0$ * Stokes}

- Modulation matrix: product of all the Mueller matrices

$$
\begin{aligned}
O & =M_{R}\left(i_{\text {Brewster }}, \lambda\right) * R(-\theta) * M_{R}(i, \lambda) * M_{R}\left(2 i-\frac{\pi}{2}, \lambda\right) * M_{R}(i, \lambda) * R(\theta) \\
& -M_{R}(i, \lambda)= \\
& \left(\begin{array}{cccc}
\boldsymbol{X}(i, \lambda)^{2}+1 & \boldsymbol{X}(i, \lambda)^{2}-1 & 0 & 0 \\
\boldsymbol{X}(i, \lambda)^{2}-1 & \boldsymbol{X}(i, \lambda)^{2}+1 & 0 & 0 \\
0 & 0 & 2 \boldsymbol{X}(i, \lambda) \cos (\tau(i, \lambda)) & 2 \boldsymbol{X}(i, \lambda) \sin (\tau(i, \lambda)) \\
0 & 0 & -2 \boldsymbol{X}(i, \lambda) \sin (\tau(i, \lambda)) & 2 \boldsymbol{X}(i, \lambda) \cos (\tau(i, \lambda))
\end{array}\right) \\
& -i \text { angle of incidence, } \lambda \text { wavelength }
\end{aligned}
$$




\section{Modulation 0: Measurements $=0$ * Stokes}

- Modulation matrix: product of all the Mueller matrices

$$
\begin{aligned}
O & =M_{R}\left(i_{\text {Brewster }}, \lambda\right) * R(-\theta) * M_{R}(i, \lambda) * M_{R}\left(2 i-\frac{\pi}{2}, \lambda\right) * M_{R}(i, \lambda) * R(\theta) \\
& -M_{R}(i, \lambda)= \\
& \left(\begin{array}{cccc}
\boldsymbol{X}(i, \lambda)^{2}+1 & \boldsymbol{X}(i, \lambda)^{2}-1 & 0 & 0 \\
\boldsymbol{X}(i, \lambda)^{2}-1 & \boldsymbol{X}(i, \lambda)^{2}+1 & 0 & 0 \\
0 & 0 & 2 \boldsymbol{X}(i, \lambda) \cos (\tau(i, \lambda)) & 2 \boldsymbol{X}(i, \lambda) \sin (\tau(i, \lambda)) \\
0 & 0 & -2 \boldsymbol{X}(i, \lambda) \sin (\tau(i, \lambda)) & 2 \boldsymbol{X}(i, \lambda) \cos (\tau(i, \lambda))
\end{array}\right) \\
& -i \text { angle of incidence, } \lambda \text { wavelength }
\end{aligned}
$$

- $\boldsymbol{X}(i, \lambda)=\frac{r_{\|}^{2}}{r_{\perp}^{2}}$ ratio of Fresnel amplitude reflection coefficients

- $\tau(i, \lambda)$ phase shift between both polarization 


\section{Modulation 0: Measurements $=0$ * Stokes}

- Modulation matrix: product of all the Mueller matrices

$$
\begin{aligned}
O & =M_{R}\left(i_{\text {Brewster }}, \lambda\right) * R(-\theta) * M_{R}(i, \lambda) * M_{R}\left(2 i-\frac{\pi}{2}, \lambda\right) * M_{R}(i, \lambda) * R(\theta) \\
& =M_{R}(i, \lambda)= \\
& \left(\begin{array}{cccc}
\boldsymbol{X}(i, \lambda)^{2}+1 & \boldsymbol{X}(i, \lambda)^{2}-1 & 0 & 0 \\
\boldsymbol{X}(i, \lambda)^{2}-1 & \boldsymbol{X}(i, \lambda)^{2}+1 & 0 & 0 \\
0 & 0 & 2 \boldsymbol{X}(i, \lambda) \cos (\tau(i, \lambda)) & 2 \boldsymbol{X}(i, \lambda) \sin (\tau(i, \lambda)) \\
0 & 0 & -2 \boldsymbol{X}(i, \lambda) \sin (\tau(i, \lambda)) & 2 \boldsymbol{X}(i, \lambda) \cos (\tau(i, \lambda))
\end{array}\right) \\
& -i \text { angle of incidence, } \lambda \text { wavelength }
\end{aligned}
$$

$X(i, \lambda)=\frac{r_{\|}^{2}}{r_{\perp}^{2}}$ ratio of Fresnel amplitude reflection coefficients

$$
\text { Depend on } \mathrm{n} \text { and } \mathrm{k}
$$

$\tau(i, \lambda)$ phase shift between both polarization

$\mathrm{n}$ : refractive index

$\mathbf{k}$ : extinction coefficient 


\section{Modulation 0: Measurements $=0$ * Stokes}

- Modulation matrix: product of all the Mueller matrices

$$
\begin{aligned}
O & =M_{R}\left(i_{\text {Brewster }}, \lambda\right) * R(-\theta) * M_{R}(i, \lambda) * M_{R}\left(2 i-\frac{\pi}{2}, \lambda\right) * M_{R}(i, \lambda) * R(\theta) \\
& =M_{R}(i, \lambda)= \\
& \left(\begin{array}{cccc}
\boldsymbol{X}(i, \lambda)^{2}+1 & \boldsymbol{X}(i, \lambda)^{2}-1 & 0 & 0 \\
\boldsymbol{X}(i, \lambda)^{2}-1 & \boldsymbol{X}(i, \lambda)^{2}+1 & 0 & 0 \\
0 & 0 & 2 \boldsymbol{X}(i, \lambda) \cos (\tau(i, \lambda)) & 2 \boldsymbol{X}(i, \lambda) \sin (\tau(i, \lambda)) \\
0 & 0 & -2 \boldsymbol{X}(i, \lambda) \sin (\tau(i, \lambda)) & 2 \boldsymbol{X}(i, \lambda) \cos (\tau(i, \lambda))
\end{array}\right) \\
& -i \text { angle of incidence, } \lambda \text { wavelength }
\end{aligned}
$$

$X(i, \lambda)=\frac{r_{\|}^{2}}{r_{\perp}^{2}}$ ratio of Fresnel amplitude reflection coefficients

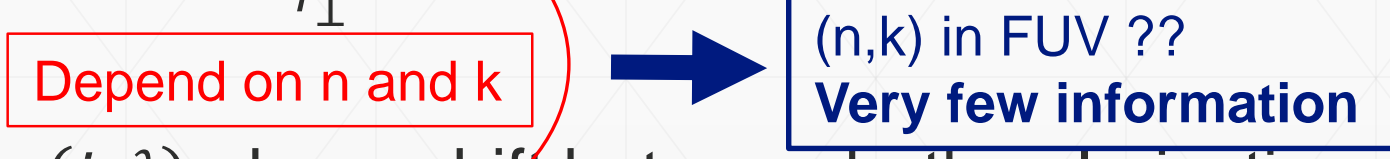

n : refractive index

k : extinction coefficient 


\section{Tests to measure $X$ and $\tau$ - use of gold}

\begin{tabular}{|c|c|}
\hline Pros & Cons \\
\cline { 1 - 1 } $\begin{array}{c}\text { Well known, even } \\
\text { at } 90 \mathrm{~nm}\end{array}$ & $\begin{array}{c}\text { Doesn't have a } \\
\text { good reflection in } \\
\text { FUV }\end{array}$ \\
\cline { 1 - 1 } $\begin{array}{c}\text { Pure : No } \\
\text { contamination }\end{array}$ & $\begin{array}{c}\text { Doesn't have } \\
\text { particular } \\
\text { polarimetric } \\
\text { properties }\end{array}$ \\
$\begin{array}{c}\text { Constant : doesn't } \\
\text { depend on the } \\
\text { supplier and the } \\
\text { process }\end{array}$ & \\
\hline
\end{tabular}

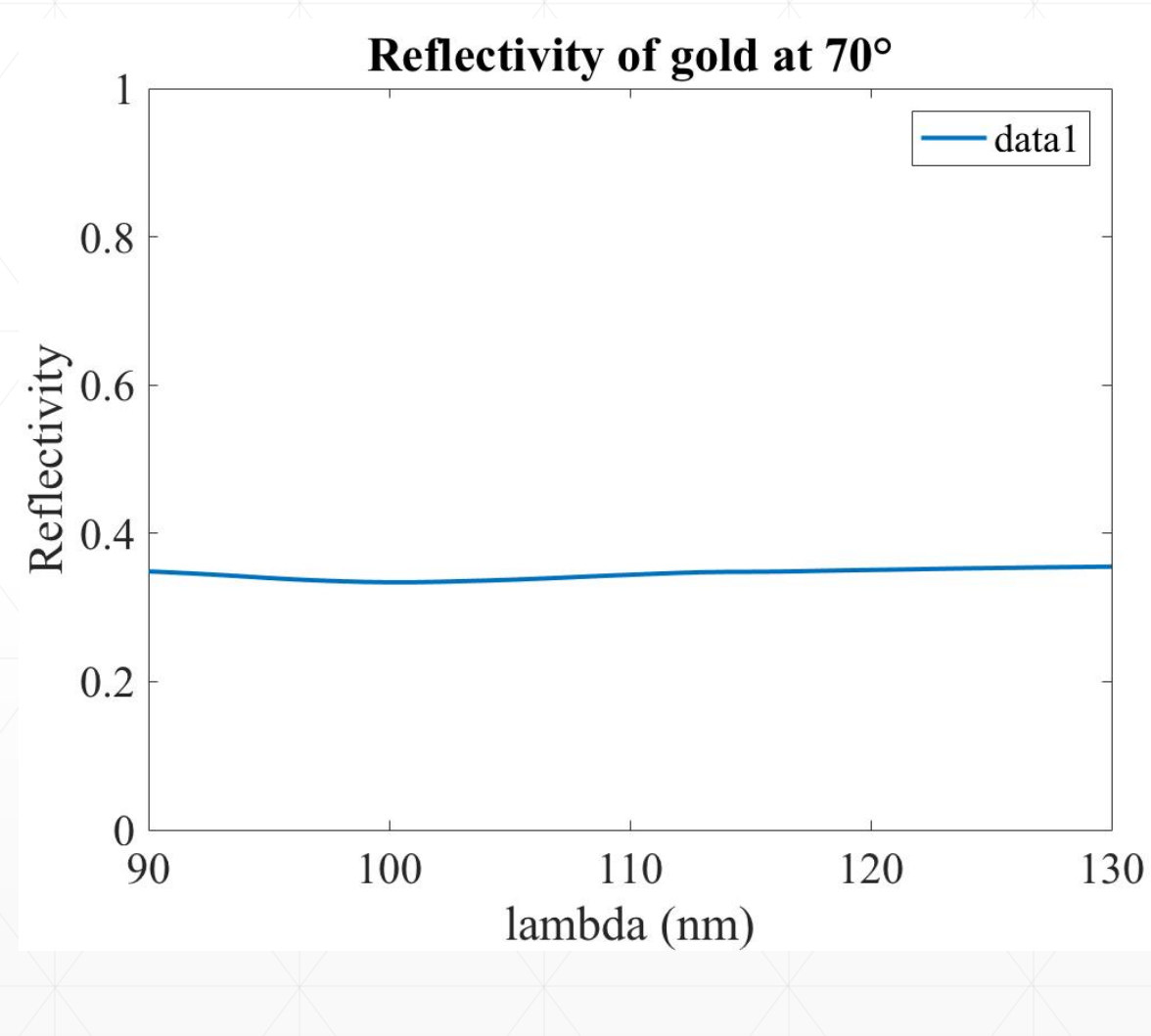




\section{Tests to measure $X$ and $\tau$}

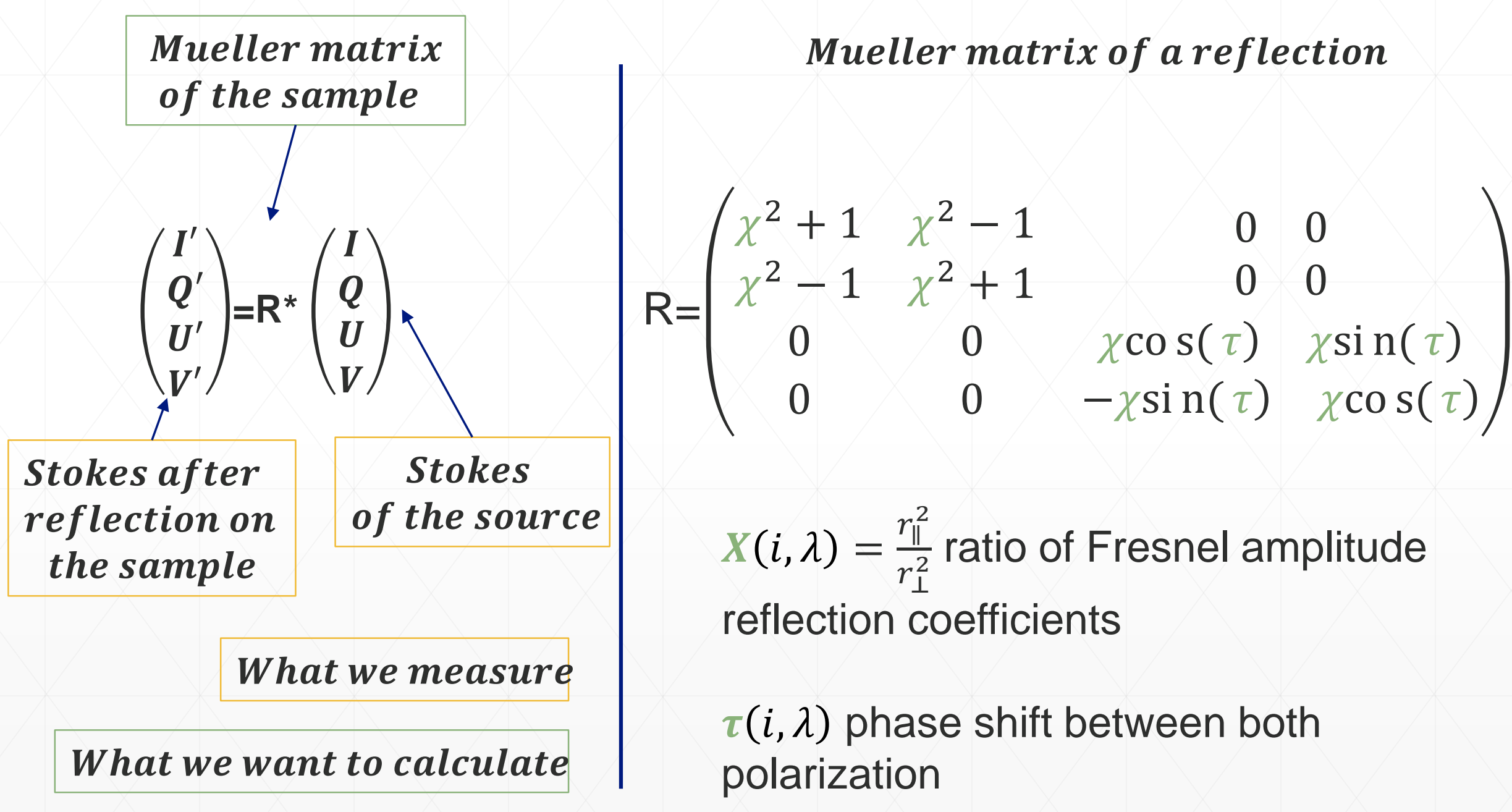




\section{Tests to measure $X$ and $\tau$ - mechanical design}

We have 3 rotations :

1. The rotation of the modulator at $180^{\circ}$ around the optical axis

2. The rotation of the sample to create different incidence angle $\rightarrow$ inclination of all the bench to follow the light

3. The rotation of the entire bench (to change the polarization of the source)

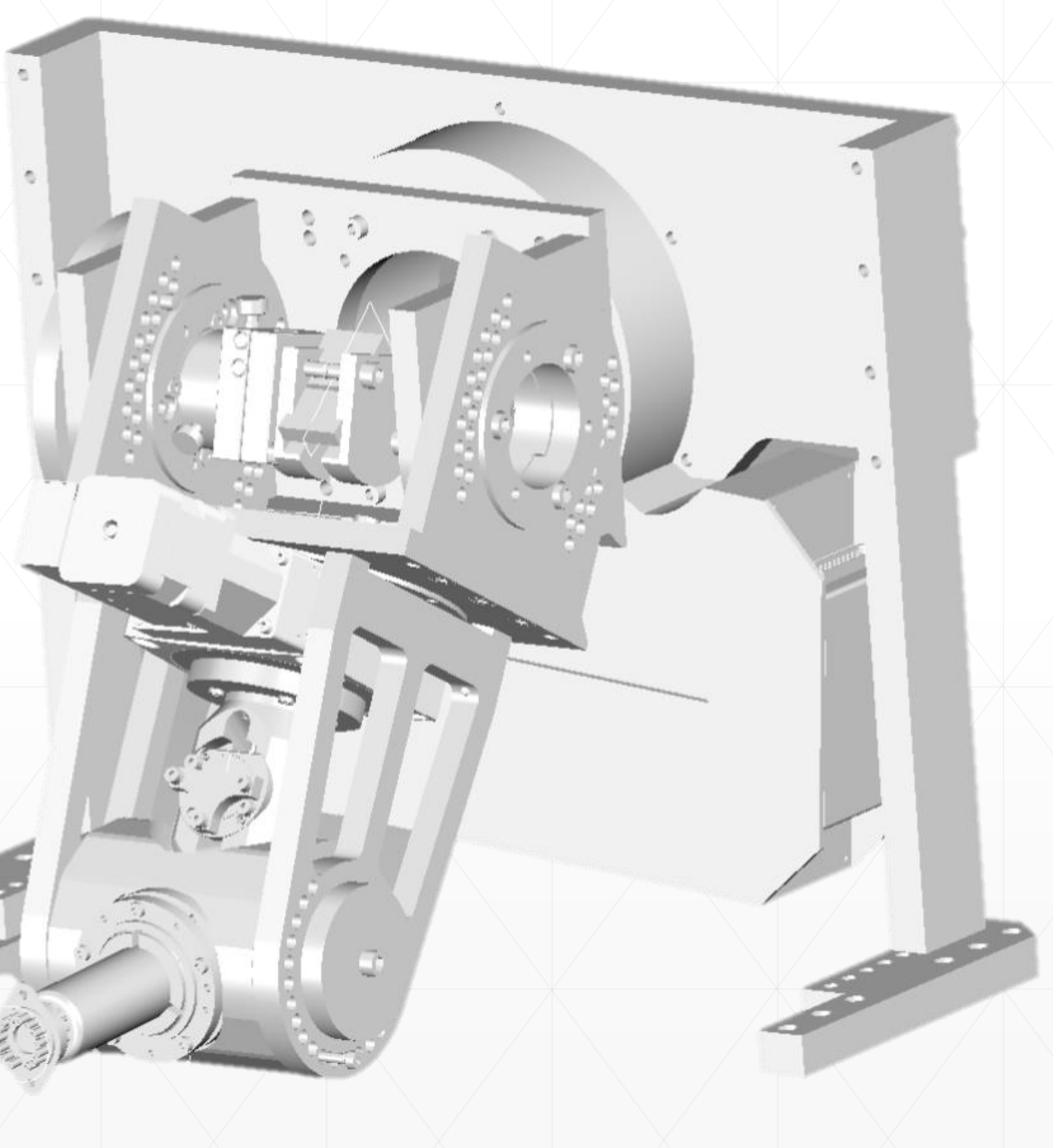




\section{Tests to measure $X$ and $\tau$ - theoretical results}

- Polarimetric efficiencies (Del Toro Iniesta et al. 2000):

$$
\epsilon_{i}=\left(n \sum_{j=1}^{n} D_{i j}^{2}\right)^{-\frac{1}{2}}
$$

- $i \in[I, Q, U, V]$,

- $n=$ number of measurements,

- $D_{i j}$ demodulation coefficients

- Optimal efficiency: $\frac{1}{\sqrt{3}} \rightarrow \mathbf{5 7} \% \quad\left(\in_{Q}^{2}+\in_{U}^{2}+\in_{V}^{2} \leq 1\right)$

- Optimization: min of

$$
\Delta \epsilon=\left|\sqrt{\left(\frac{1}{\sqrt{3}}-\epsilon_{Q}\right)^{2}+\left(\frac{1}{\sqrt{3}}-\epsilon_{U}\right)^{2}+\left(\frac{1}{\sqrt{3}}-\epsilon_{V}\right)^{2}}\right|_{\lambda}
$$




\section{Tests to measure $X$ and $\tau$-theoretical results}

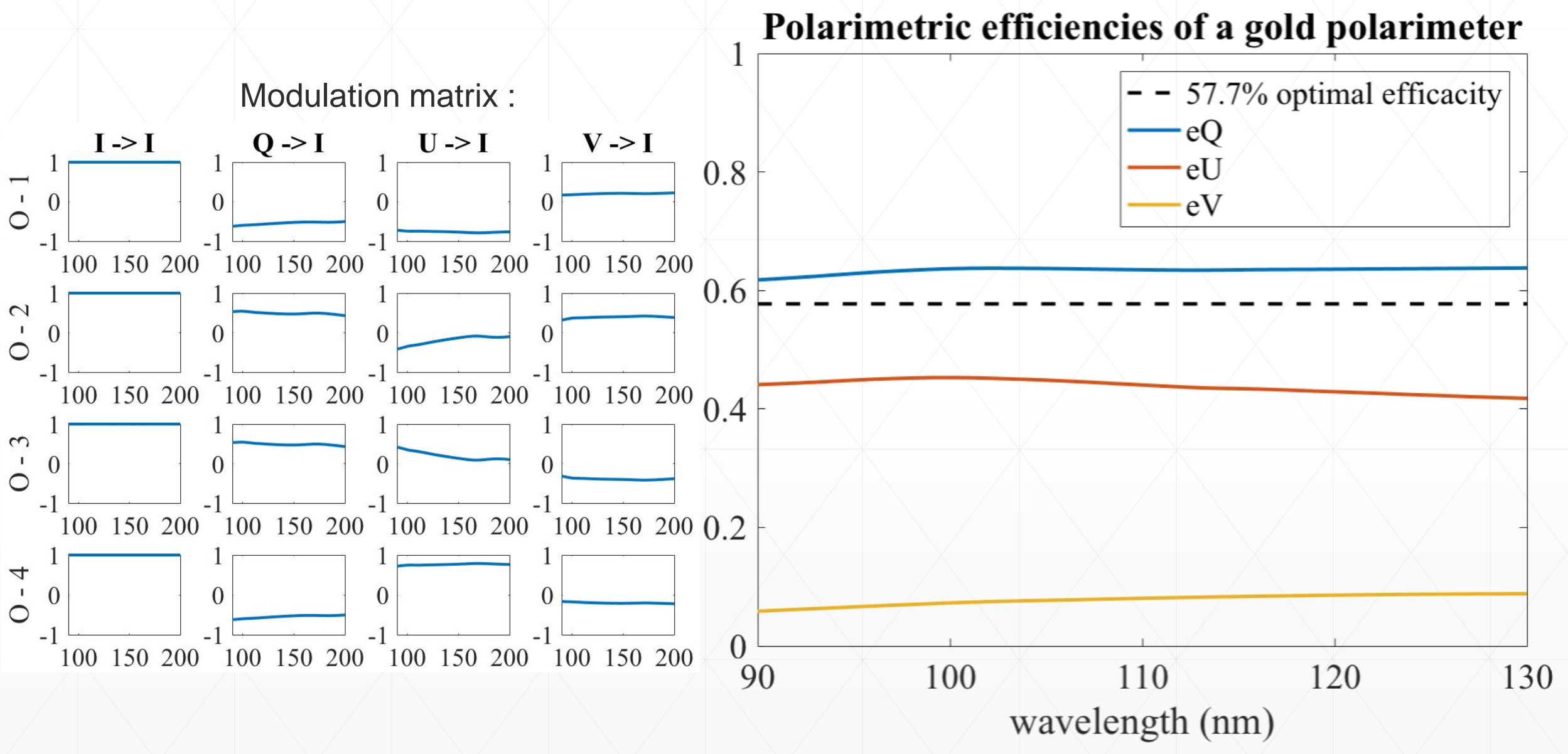




\section{Tests to measure $X$ and $\tau$ - theoretical results}

Quasi

Brewster angle?

Contrast as a function of wavelength and incidence angle

Best contrast between $p$ and $s$ polarization at $\mathrm{i}=61^{\circ}$

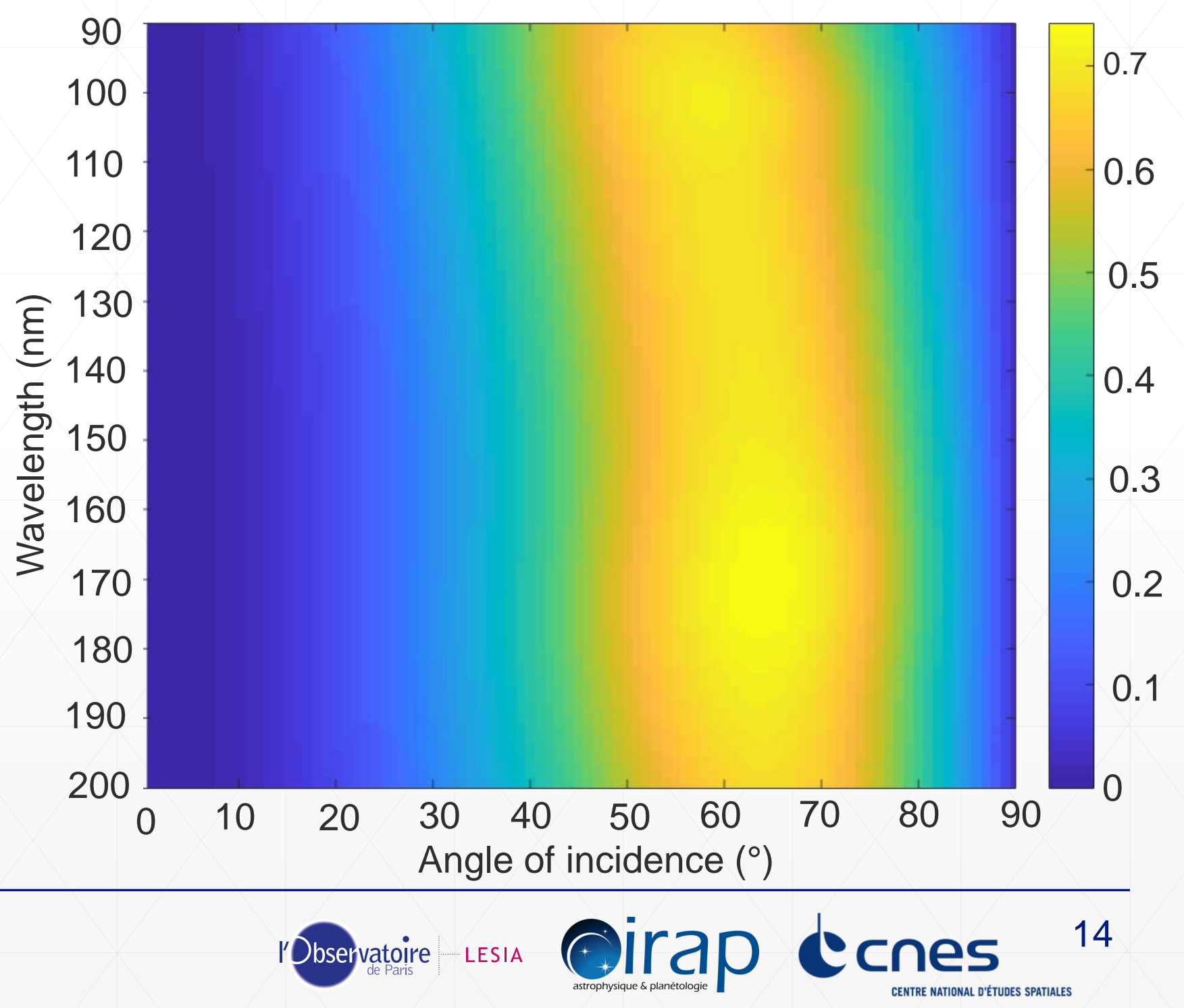




\section{Tests to measure $X$ and $\tau$ - theoretical results}

Figure of merit of the polarizer: $\varepsilon=C \sqrt{\frac{R_{S}+R_{P}}{2}}$

For a perfect polarizer, figure of merit at 0.71

Figure of merit of a gold polarizer at $61^{\circ}$

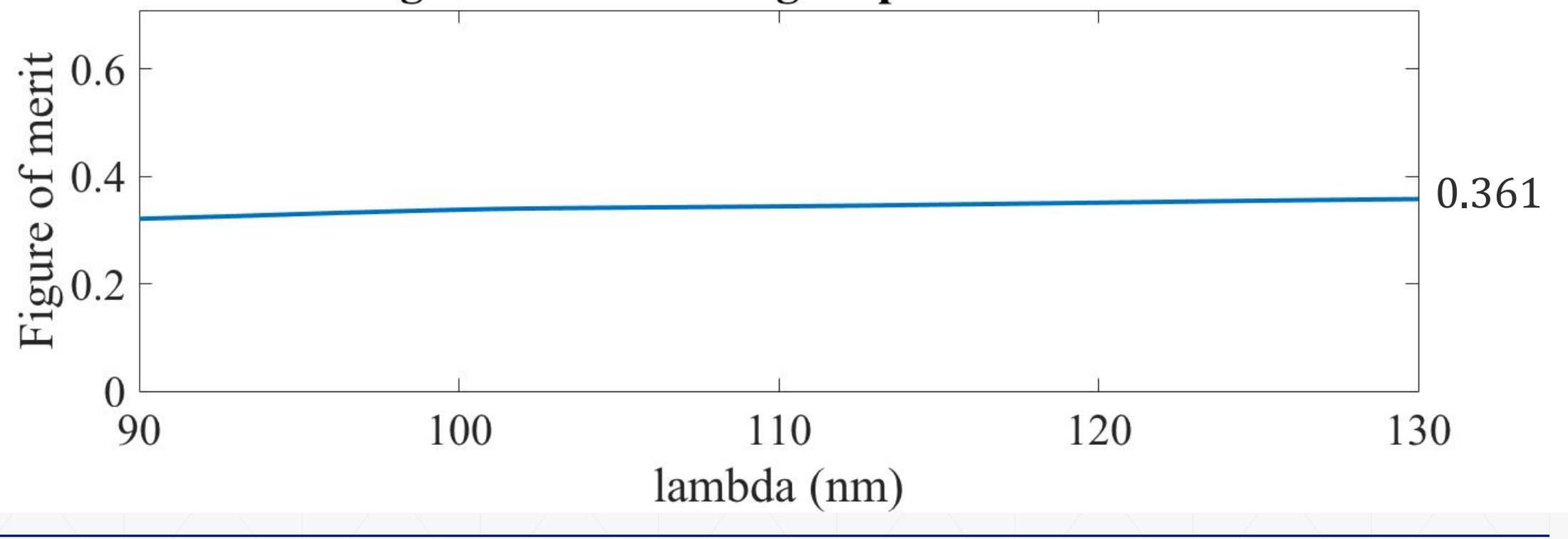




\section{Tests to measure $X$ and $\tau$ - planning}

- Design of the polarimeter $: \checkmark$

- First definition of the test procedure $: \checkmark$

- Optomechanical design of the test setup : $\checkmark$

- Manufacture of the mechanical pieces: on-going

- Installation of the setup: October 2018

- Commissioning: November 2018

- Start of the measurement: November 2018 
- A new type of polarimeter has been studied for the FUV in the frame of the Pollux study for LUVOIR.

- A first polarimeter using gold has been studied (modulation, transmission, efficiencies) opening the way for future FUV polarimeters.

\title{
Conclusion
}

- An experiment is being installed to choose the best material and optimize the polarimeter.

I'Observatoire LESIA

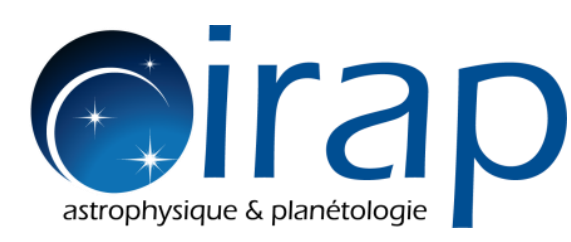

\author{
Thank you! \\ Questions ?
}

